\title{
INFORMATION SUPPORT OF HEALTH-RELATED QUALITY OF LIFE OF PATIENTS WITH OCCUPATIONAL DISEASES
}

\author{
M.P. Diakovich \\ Irkutsk scientific center \\ Angarsk State Technical University \\ East-Siberian Institute of medical and ecological researches \\ Angarsk, Russia \\ marik914@rambler.ru \\ I.A. Finogenko \\ Irkutsk scientific center \\ Matrosov Institute of System Dynamics and Control Theory \\ Irkutsk, Russia \\ fin@icc.ru \\ A.A. Blokhin \\ Irkutsk scientific center \\ East-Siberian Institute of medical and ecological researches \\ Angarsk, Russia \\ senyadiamond@yandex.ru
}

\begin{abstract}
The aim of this study is to develop information support of research of health-related quality of life (HRQoL) of patients with occupational diseases in multicenter studies. The authors have developed an automated system, which facilitates to collect and process data in multicenter trials. The automated system has used to assess HRQoL of patients with occupational diseases taking into account socio-economic characteristics of the regions of their residence at some point of time or in dynamics of development of chronic diseases research. Automated use of the analytic hierarchy process in the system analysis of HRQoL of patients with occupational diseases, being an extremely complex object with lots of multidimensional, heterogeneous relationships, allows us to estimate the degree of influence of individual components of the object as a whole. This study highlights the importance of developing the automated system, which significantly facilitates the work of researchers on the collection and data processing in multicenter studies and automated use of analytic hierarchy process on the system analysis of HRQOL of patients with occupational diseases. These studies go further to solving HRQOL control tasks.
\end{abstract}

Keywords - health-related quality of life, patients with occupational diseases, multicenter studies, information support, automated system, analytic hierarchy process

\section{INTRODUCTION}

Both scientists and politicians are considering the quality of life of the population as a criterion for the efficiency of the separate subsystems of society. Currently aspects directly related to human health and the health system have been allocated from this general concept. The World Health Organization defines quality of life as an individual assessment of their social status in the context of the culture of this society in view of the plans, opportunities, standards and interests. In spite of the fact that the Ministry of Health of the Russian Federation declared the concept of study of health-related quality of life (HRQoL) in medicine as a priority as far back as 2001, this concept has not been widely used in Russia until now.

Research carried out in the framework of the integration program of the Irkutsk Scientific Center of SB RAS "Fundamental research and breakthrough technology as the basis for the advanced development of the Baikal region and its interregional relations" project 3.4, and with partial financial support of RFBR grant №14-07-00116
Knowledge about HRQOL could significantly supplement the existing standards of examination of a patient with any disease. Objective medical information, supplemented by subjective data about the attitude of the patient to his overall health and functional capabilities, allows us to give a more precise picture of the health of the patient. At the same time, objective indices registered by instrumental methods are often more variable than subjective ones [1].

The morbidity of the working population caused by the influence of occupational factors entails significant economic damage and, therefore, poses a serious problem for Russia today. It should be noted that occupational pathology becomes largest value in medium-term prospect, identifying trends in the development of economic, industrial and demographic development of the country.

The working conditions at $73.6 \%$ of Russia's industrial enterprises have determined the risk of occupational diseases among workers according to the data from the Federal Service for Supervision of Consumer Rights Protection and Human Wellbeing in 2014. To the greatest extent this problem is typical for areas with a high share of the population working in the industrial sector, and especially in Siberia, which is the economic and industrial potential of the country. The indicators of occupational diseases in the Irkutsk region during 20122014 ranged from 4.01 to 5.02 per 10,000 employees, and they are 2.4-2.9 times exceeding the nationwide indicators [2].

The occurrence of a monthly allowance from the state, a low level of medical and social rehabilitation, non-compliance of patients with occupational diseases $[3,4]$ in one hand, and the paucity of quality of life research in occupational pathology $[5,6,7]$ on the other hand determine the relevance of our research.

Multi-center study, conducted by unified program simultaneously in specialized medical establishments in different territories, allow getting information about the territorial and professional features of HRQoL and reducing the time of collecting the required amounts of information to obtain reliable results.

The specificity multi-center study is caused by the geographical remoteness of research and the complexity of the 
organization of information exchange between them [8]. Due to the large quantities of data in studies of this kind, as well as to solve logistical problems and the organization of an effective informational exchange, the use of automated systems for the collection, processing, storage, analysis and presentation of data on the HRQoL of patients participating in the multi-center study is actual.

Not numerous automated systems $[9,10]$ both in Russia and abroad are more focused on the managerial sector, they are not intended for application in the multi-center study, using the author's methodology for assessing HRQoL, that does not allow to perform comparative analysis with other research.

\section{RESULTS AND DISCUSSION}

The study of HRQoL of patients with occupational diseases sets two serious problems in front of researchers. The first task is to collect information about the HRQoL of patients with various forms of occupational diseases in different territories, reflecting the objective indices of functioning of all regional areas of life and subjective assessment of life satisfaction. The second problem is HRQoL of patients control task. The solution to this problem is not possible without the creation of mathematical and informational models of such complicated system.

Analysis of process of multicenter studies and modern information technology capabilities allowed us to offer a new scheme of information support of research HRQoL of patients with occupational diseases. As a central unit that performs the basic work on the organization of information flows automated system for the collection, validation and processing of data obtained directly from the patients has been offered. The main functional blocks of the system are represented by relational database for storage of responses, results of data processing and professional, and demographic characteristics of patients; subsystem for calculation the main parameters on the techniques of research; user interface for data entry and checking them for correctness. The proposed scheme allows patients to get rid of errors in filling out questionnaires, the researchers - to get rid of errors in manual processing of the questionnaires. This system allows significantly speed up and simplify the process of interaction between research centers in multicenter study. Moreover, the patient will be able to of operational awareness about the individual characteristics of the HRQL, and leader of the study will be able to audit as a researcher and research center, calculate basic statistical parameters for samples of patients. However, the commissioning of such a system will require additional time and financial resources. However, if the system will be expandable with the functional integration to new research, these deficiencies in the system will become insignificant.

\section{A. Work accomplished}

Analysis of the requirements for such an automated system and development tools meet these requirements, database management systems and design patterns was conducted. The most appropriate to the specifics of the system being developed from the point of view of a client-server architecture of the automated system were found the database management systems PostgreSQL, Python programming language, Django framework and development environment PyCharm JatBrains.
The results of the development and design of the system the web- server has been created, which allows you to organize the process of remote information gathering both from patients and from researchers via the Internet.

In addition to SF-36 questionnaire recommended by World Health Organization and adapted for use in the Russian experts Multinational Research Center of quality of life in medicine (SPb., 1999), 11 techniques have been integrated additionally into the developed software tool. These techniques showed themselves to advantage in ensuring the collection of objective information about the general state of physical, socio-demographic and psychological profile of patients with occupational diseases [11]. The introduction of additional techniques clarifies assessment of HRQoL and expands the range of possible research activities.

To protect the personal data of patients the process of authorization/authentication is provided in automated system, as well as a set of groups, the rights and permissions; in addition, required database encryption is also provided. There is a possibility of anonymization of examined patients. Personal name/password is provided for researchers and assistants of each study center, a responsible person registers them in the system. Access to view and records edition are allowed to researchers and assistants only for patients who were examined by their. The person responsible for the research center has access to the profiles of the surveyed in this institution only. The function of data collection audit in research centers is very important. Automated system administrators have access to data on the number of surveys by separate techniques and the number of completed surveys. There is the ability to analyze information gathered by separate investigator and a separate research center.

The process of examination of the patient consists in its registration and testing directly on-line. If a patient accidentally skips a question in the survey, automated system reminds him about it before saving the results. After the completion of session automated system shows the results of the survey to the patient and the investigator. At that, each indicator corresponds to a comment that describes its overrun beyond the reference values. There is a possibility of monitoring individual performance, if the patient has previously carried out a survey on the techniques presented in automated system.

\section{B. Work in progress}

The solving HRQoL control tasks require analysis of its structure. The number of these elements and relationships may be so high that it exceeds the capacity of researchers to receive the information in its entirety. Therefore, the construction of hierarchy, which reproduces the functional relationship must be preceded to the analysis of HRQoL system functioning.

Successful use of analytic hierarchy process by T. Saati in technology and economy has prompted us to use it as a tool for qualitative analysis and numerical ranking criteria HRQOL as an object with a large number of diverse factors with varying degrees of importance.

The first step in the application of analytic hierarchy process is decomposition and construction of hierarchical 
structural model of the system, wherein indicators of HRQoL are distributed through the levels, and the higher level elements are dependent on the downstream elements. Elements in groups of the same level do not depend on each other, but in transitions to other levels they can enter into certain relationships with each other.

The second step in the application of analytic hierarchy process consists in construction the matrix of pairwise comparisons for each group of criteria, which are compared in pairs in relation to their common components of a higher level of the hierarchy. Since a person acting as an expert, has the psychological limit of perception, simultaneous comparison of no more than 9 criteria is considered acceptable. So, ninepoint scale is used for subjective pairwise comparisons.

The third step in the application of analytic hierarchy process is to obtain the weight coefficients being characteristics for each quantitative criterion of HRQoL. It should be noted that the ratio of weight coefficients are present in the matrix of pairwise comparisons, but the values of these coefficients are not known in advance and must be determined by mathematical methods.

The fourth step in the application of analytic hierarchy process is to calculate the consistency ratio for the matrices of pairwise comparisons. Consistency ratio is a measure of the accuracy of the pairwise comparisons in the second step. Consistency ratio is equal to zero in the ideal case of completely agreed back symmetric matrix of pairwise. The problem is to determine the effect of the elements of the lower levels to the upper level (the integral index HRQoL) through intermediate levels. HRQoL scales indicators used by us to build its four-level hierarchical model reflecting all the functional relationship between the elements, which are combined in groups and are distributed through the levels of the hierarchy [12]. The first level is the integral index of HRQoL - purpose of the study.

Computational part of the method is simple and can easily be algorithmic. Pairwise comparison of HRQoL indicators (basis for calculation procedures of analytic hierarchy process) requires informal participation of experts and can be automated only partially. For information support of analytic hierarchy process, the software in the environment of Delphi object-oriented programming (version Turbo Delphi Explorer), which is based on ObjectPascal language developed in his graduate qualification work of the bachelor D. Zhernov under the guidance I. Finogenko. Delphi development environment allows to quickly create applications, produces small in size high efficiency executable modules, offers high performance and has low requirements application under development to computer resources. Information support is provided by simple, convenient and easy to learn interface, which provides the end user all the necessary functional for his work. This interface provides the ability to input information about the HRQoL of patients surveyed, automated counting of output data, comparing the output data and storing them in the form of diagrams and tables for further comparative analysis.

\section{Future work}

It is expected to further expand system capabilities. Currently, there is a possibility to carry out multi-center study using the techniques from the existing set in the system. In the future, the researchers will be able to use the automated system as a "designer" of multi-center study. If researchers want to use more techniques to assess HRQoL, it will be possible to integrate the missing tools in the system before carrying out a new study.

In the future, it is believed possible to use the priorities identified by using analytic hierarchy process, for setting and solving the optimization problem HRQoL and the development information support of making management decisions on these priorities for improving the quality of life of patients.

\section{CONCLUSION}

Thus, we have developed the automated system, which significantly facilitates the work of researchers on the collection and data processing in multi-center study. Automated system is used to measure HRQoL of patients with occupational diseases, taking into account the socio-economic characteristics of the regions where they lives, it allows researchers to assess HRQoL at any given time and in the dynamics of the development of chronic diseases. Thanks to the authors' system, it becomes possible to the competent organization of business processes of multicenter study, simplified preparation for the study, and the data collection process itself.

Analytic hierarchy process is used for numerical comparison of the criteria studied object, for comparison of alternatives and selects the best solution from a set of possible or proposed. Thus, analytic hierarchy process can be used to study HRQoL. Automated use of analytic hierarchy process on the system analysis of HRQoL of patients with the occupational diseases, which is an extremely complex subject with an abundance of multi-dimensional, heterogeneous relationships, allows evaluating the influence of some of its components to the object as a whole.

The results of the application of analytic hierarchy process allow setting and solving the optimization problem HRQoL of patients with occupational diseases on the identified priorities. This will have a significant social effect because it would facilitate decision-making stakeholders for improving the quality of life of patients.

\section{REFERENCES}

[1] Health related quality of life: assessment and management, V. Rukavishnikov, eds. Irkutsk: NC PBX, 2012, 168p.

[2] On the state sanitary and epidemiological welfare of the population in the Russian Federation in 2014: State Report. Moscow: Federal Service for Supervision of Consumer Rights Protection and Human Welfare, 2015, pp.74-92.

[3] M.P. Dyakovich, T.V. Kazakova, I.Y. Solovyova Complex assessment of quality of life as an indicator of the effectiveness of medical, social and professional rehabilitation of persons with disabilities as a result of mercury neurotoxicity, Moscow, Health of the Russian Federation, 2012, vol. 6, pp. 35-38.

[4] Z.M. Berheeva, N.V. Linkov, O.A. Ishteryakova, I. Yu. Malyshev, N.I Novikov, L.V. Shakirov Modern medical-social examination and rehabilitation in occupational diseases, Kazan, Kazan Medical Journal, 2003, vol.3, pp. 221-224. [5] M.P. Dyakovich, V.A. Semenihin, P.V. Kazakova, O.V. Odintsova, O.A . Dyakovich The quality of life of persons with occupational diseases from exposure to various industrial 
factors, Moscow, Occupational Medicine and Industrial Ecology, 2014,vol.2, pp. 27-32.

[5] Chetukova DH, Savin AA An influence of vibration disease on quality of life of workers in industry and agriculture, Kazan, Kazan Medical Journal, 2013, vol. 4, pp.483-487.

[6] D. Boehm, G. Schmid-Ott, F. Finkeldey, S.M. John, C. Dwinger, T. Werfel, T.L. Diepgen, K. Breuer. Anxiety, depression and impaired health-related quality of life in patients with occupational hand eczema, Copenhagen: Munksgaard, Contact Dermatitis, 2012, vol. 67, issue 4, pp.184-192

[7] A.A. Novik, T.I. Ionova Guide to the study of quality of life in medicine 2nd ed., Moscow: Olma Media Grupp, 2007, 320 p.

[8] E.P. Wright, P.J. Selby, M. Crawford Feasibility and compliance of automated measurement of quality of life in oncology practice , Journal of clinical oncology, 2003, vol 2,. pp. 374-382.

[9] Y.I. Shtevnina, S.L. Shvyrev, T.V. Zarubina, I.V. Shirokorad, D.V. Dolgih Development and implementation of an automated evaluation of the quality of life of cancer //Medical Technology. Evaluation and selection, 2012, vol. 2, pp. 106-113.

[10] M.P. Dyakovich, P.V. Kazakova Organization of research on complex assessment of the quality of life of persons with occupational pathology, Guidelines, approved by the Scientific Council of №45 of the Russian Academy of Medical Sciences and Health and Social Development of the Russian Federation for medical and environmental, workers' health problems. Irkutsk: NCRS SB RAMS, 2013, 56p.

[11] I.A. Finogenko, M.P. Dyakovich, A.A. Blokhin The methodology of evaluation of quality of life related to health. Vestnik Tambov University. Ser. Natural and Technical Sciences. 2016. vol. 21, Issue 1, pp.

121-130 
